\title{
Renormalization Group Analysis of Quasi-Periodicity in Analytic Maps ${ }^{\star}$
}

\author{
Michael Widom ${ }^{\star \star}$ \\ The James Franck Institute and The Department of Physics, The University of Chicago, Chicago, \\ IL 60637, USA
}

\begin{abstract}
Analytic maps of the form $f(z)=e^{2 \pi i \Omega} z+\mathcal{O}\left(z^{2}\right)$ display quasiperiodicity when $\Omega$ satisfies a diophantine condition. Quasiperiodic motion is confined to a neighborhood of the origin known as a Siegel domain. The boundary of this domain obeys universal scaling relations. In this paper we investigate these scaling relations through a renormalization group analysis, and we discuss singularities and asymptotic form of the scaling function.
\end{abstract}

\section{Introduction}

The discovery of scaling laws associated with the transition from quasiperiodic to chaotic behavior is an exciting development in the theory of dynamical systems. Shenker and Kadanoff [1], following Greene's [2] methodology, found that K.A.M. tori in area preserving maps disappear by becoming nondifferentiable in a scale invariant fashion. Shenker [3] studied the analogous phenomenon in dissipative systems and found scaling behavior in maps of a circle.

These transitions lie in universality classes; many different mappings show identical scaling behavior. Thus one can use simple models to analyze the scaling behavior of complicated dynamical systems. Provided a dynamical system satisfies a few constraints, the scaling laws are independent of all other details. Renormalization group arguments, introduced into the theory of mappings by Feigenbaum [4], explain this universality. Collet and Eckmann [5] discussed this application of the renormalization group.

In a previous application of the renormalization group to quasiperiodic systems, Kadanoff $[6,7]$ explored universal scaling functions for K.A.M. tori. Escande and Doveil [8] implemented the renormalization group on Hamiltonians instead of maps. MacKay [9] obtained a numerical solution of the renormal-

\footnotetext{
* A thesis submitted to the Department of Physics, University of Chicago, Chicago, Illinois, in partial fulfillment of the requirements for the Ph.D. degree

$\star \star$ Present address: Department of Physics, Harvard University, Cambridge, MA 02138, USA
} 
ization group equations for area preserving maps. Feigenbaum et al. [10] and Rand et al. [11] solved the renormalization group equations for circle maps.

In this paper we employ renormalization group techniques to study the boundaries of Siegel domains $[12,13]$ in analytic maps. Siegel domains occur in maps of the form $f(z)=e^{2 \pi i \Omega} z+\mathcal{O}\left(z^{2}\right)$ for special values of $\Omega$. Inside the Siegel domain trajectories execute quasiperiodic motion on "Siegel curves" which are analogous to K.A.M. tori in area preserving maps. Manton and Nauenberg [14] found that the boundary of the Siegel domain is nondifferentiable and obeys a universal scaling law similar to that obeyed by critical K.A.M. tori. Section 1 of this paper describes Siegel curves and the scaling laws they obey. In Sect. 2 we introduce the renormalization group, derive a set of equations which the scaling function must obey, and solve these equations numerically. In Sect. 3 we linearize the renormalization group equations to explore universality. Finally, in Sect. 4 we examine a family of singularities in the universal scaling function and address the question [15]: Is the critical point really on the boundary of the Siegel domain? We derive an analytic expression for the universal scaling function which should be valid only if the critical point lies on the boundary of the Siegel domain, and find that the scaling function fits this expression with great accuracy.

\section{Siegel Curves and Scaling}

Siegel considered mappings of the complex plane of the form

$$
z^{\prime}=f(z)=e^{2 \pi i \Omega} z+\sum_{n=2}^{\infty} a_{n} z^{n}
$$

where the sum in Eq. (1.1) converges to an analytic function. Siegel proved that when $\Omega$ satisfies the diophantine condition

$$
|\Omega-m / n|>\lambda n^{-\mu}
$$

for all integers $m, n \geqq 1$, and some $\lambda, \mu>0$, there is a neighborhood $U$ containing the origin in which $f$ is conjugate to a pure rotation. This means there is a coordinate transformation $z=\phi(w)$, smooth and invertible for $z \in U$, which transforms $f$ into multiplication by $e^{2 \pi i \Omega}$, i.e.:

$$
f(\phi(w))=\phi\left(e^{2 \pi i \Omega} w\right) .
$$

Hence, in terms of the variable $w$,

$$
w^{\prime}=\phi^{-1} \circ f \circ \phi(w)=e^{2 \pi i \Omega} w .
$$

Equation (1.3) is known as the Schröder equation [16].

When $z_{0} \in U$ we can use Eqs. (1.3) and (1.4) to simplify the process of iterating the mapping $f$. If we write

$$
z_{n}=f^{(n)}\left(z_{0}\right)=\underbrace{f \circ f \circ \ldots \circ f}_{n \text {-times }}\left(z_{0}\right),
$$




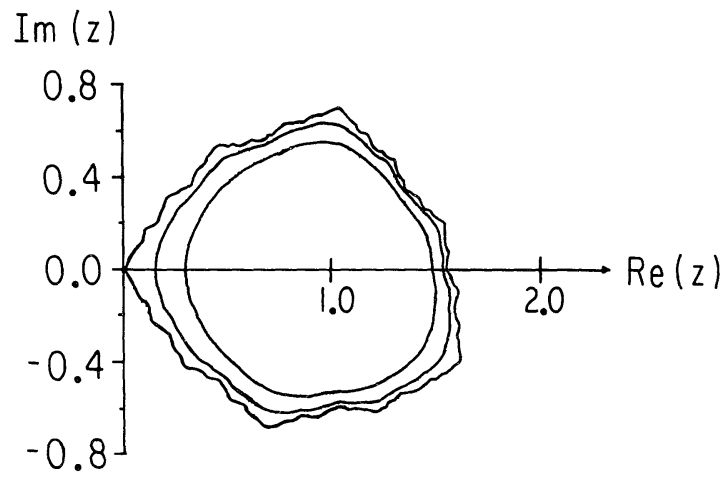

Fig. 1. Trajectories of a quadratic mapping with $f(1)=1, f^{\prime}(1)=e^{2 \pi i \Omega}$, and $f^{\prime}(0)=0$. The bumpy curve is generated by the critical point $z_{c}=0$

and define $w_{n}$ by

$$
z_{n}=\phi\left(w_{n}\right)
$$

then by Eq. (1.4)

$$
w_{n}=e^{2 \pi i n \Omega} w_{0} .
$$

The $w_{n}$ densely fill the circle $\left\{w:|w|=\left|w_{0}\right|\right\}$, so the $z_{n}$ densely fill the image of this circle under $\phi$. This image is a smooth, closed, invariant curve of $f$ known as a Siegel curve. The neighborhood $U$ is known as a Siegel domain. Every point in the Siegel domain lies on a Siegel curve.

Siegel curves have a winding number $W=\Omega$. To see this consider the rotation in the $w$-plane. After $n$ iterations the phase of $w$ increases by $2 \pi n \Omega$. Thus we can think of the trajectory $\left\{w_{k}: k=0,1, \ldots, n\right\}$ as having wound around the origin

$$
m(n)=[n \Omega]
$$

times, where $[a]$ denotes the integer part of $a$. We define the winding number $W$ as the average number of rotations per iteration, thus

$$
W=\lim _{n \rightarrow \infty} \frac{m(n)}{n} .
$$

From Eq. (1.8) we see that the winding number in the $w$-plane is just $\Omega$. By the conjugacy $\phi$ we find that in the $z$-plane also we have

$$
W=\Omega \text {. }
$$

Manton and Nauenberg studied the boundary $\partial U$ at which $\phi^{-1}$ ceases to exist (see Fig. 1). They found that for a class of mappings of the form (1.1), $\partial U$ has the following properties :

1) $\partial U$ is a Siegel curve with winding number $\Omega$.

2) $\partial U$ is nowhere differentiable.

3) $\partial U$ has universal scale invariant structure.

4) $z_{c} \in \partial U$, 
where $z_{c}$ is the critical point of $f\left(f^{\prime}\left(z_{c}\right)=0\right)$ closest to the origin. For the remainder of this section we discuss the scale invariance of $\partial U$. We address the observation $z_{c} \in \partial U$ in Sect. 4 .

Smooth curves in the interior $U$ possess a trivial scaling simply related to the winding number $\Omega$. We choose the value $\Omega=\frac{\sqrt{5}-1}{2}$ because it is especially simple to analyze. $\Omega$ satisfies the inequality

$$
|\Omega-m / n|>\frac{1 / \sqrt{5}}{n^{2}}
$$

We analyze trajectories contained in $U$ in the $w$-plane instead of the $z$-plane. Consider the Fibonacci iterates $z_{Q_{N}}=f^{\left(Q_{N}\right)}\left(z_{0}\right)$ of a point $z_{0} \in U$, where the $N^{\text {th }}$ Fibonacci number $Q_{N}$ is defined by

$$
Q_{N}=Q_{N-2}+Q_{N-1}=Q_{N-1}+Q_{N-2},
$$

and the initial conditions

$$
Q_{0}=0, \quad Q_{1}=1
$$

Fibonacci numbers satisfy

$$
Q_{N} \Omega=Q_{N-1}-(-\Omega)^{N}
$$

Thus for large $N$

$$
w_{Q_{N}} \sim w_{0}\left(1-2 \pi i(-\Omega)^{N}\right)
$$

or, inside the Siegel domain in the $z$-plane,

$$
z_{Q_{N}} \sim z_{0}+C(-\Omega)^{N}, \quad C=-2 \pi i w_{0} \phi^{\prime}\left(w_{0}\right) .
$$

Equation (1.16) is the trivial scaling for smooth invariant curves in the interior of $U$. Fibonacci iterates approach the starting point $z_{0}$ at the rate $\beta=\Omega^{-1}$. Note that $\beta$ is universal. Provided that $z_{0} \in U, \beta$ depends on no details of $f$ other than $\Omega$. Equation (1.16) cannot be applied for $z_{0} \in \partial U$ because $\phi^{-1}$ is nondifferentiable on $\partial U$.

The nontrivial scaling associated with $\partial U$ found by Manton and Nauenberg resembles Eq. (1.16). They found that for functions of the form

$$
f(z)=e^{2 \pi i \Omega} z+z^{2}+\lambda z^{3}
$$

the scaling law takes the form

$$
z_{Q_{N}}=f^{\left(Q_{N}\right)}\left(z_{c}\right)=z_{c}+c(\lambda) \alpha^{-N} e^{i \theta_{N}}
$$
where $\alpha=1.34783$ and $\theta \equiv\left|\frac{\theta_{N+1}-\theta_{N}}{2}\right|=0.93611$ are independent of $\lambda$ for all but
isolated values of $\lambda$.

Equations (1.16) and (1.18) can assume a form which suggests more general scaling properties. First translate coordinates so that the starting point is at the origin, then rotate so that $\theta_{N+1}=-\theta_{N}$. Equations (1.16) and (1.18) take on the 
standard form

$$
\begin{gathered}
z_{Q_{N}} \sim \beta^{-N} e^{ \pm i \pi / 2}, \\
z_{Q_{N}} \sim \alpha^{-N} e^{ \pm i \theta}
\end{gathered}
$$

where the $+(-)$ sign holds for $N$ even (odd). Defining $f_{N}(z) \equiv f^{\left(Q_{N}\right)}(z)$ and noting $z_{Q_{N}}=f_{N}(0)$, we obtain

$$
\begin{gathered}
f_{N}(0) \sim \beta^{-N} e^{ \pm i \pi / 2} \\
f_{N}(0) \sim \alpha^{-N} e^{ \pm i \theta}
\end{gathered}
$$

We can try to generalize Eqs. (1.16b) and (1.18b) to include nonzero values of the argument $z$. From the work of Manton and Nauenberg it appears that the limits

$$
\begin{aligned}
& \lim _{N \rightarrow \infty} \beta^{N} f_{N}^{* N}\left(\beta^{-N} z\right)=F_{i}(z), \\
& \lim _{N \rightarrow \infty} \alpha^{N} f_{N}^{* N}\left(\alpha^{-N} z\right)=F_{c}(z),
\end{aligned}
$$

exist and that $F_{i}$ and $F_{c}$ are universal functions associated, respectively, with the interior of $U$ and the critical point $z_{c}$. The values of $F_{i}(0)$ and $F_{c}(0)$ are $e^{i \pi / 2}$ and $e^{i \theta}$. We define the operation * by

$$
f^{*}(z)=\bar{f}(\bar{z})
$$

where "-" denotes conjugation, and $*^{N}$ denotes * performed $N$ times. Note that $*^{N}$ is the identity when $N$ is even, and that $F^{*}$ is analytic if $F$ is analytic. In the next section we employ renormalization group techniques to investigate the validity of Eqs. (1.16c) and (1.18c).

\section{Renormalization Group}

The renormalization group is a set of transformations of a space of functions onto itself. We define a sequence of transformations with the universal functions $F_{i}$ and $F_{c}$ as fixed points. A preliminary transformation translates the origin to some point $z_{0}$ we wish to study. If $z_{0} \in U$, then our subsequent transformations will generate $F_{i}(z)$. If $z_{0}=z_{c}$, then our subsequent transformations will generate $F_{c}(z)$. Define

$$
g_{1}(z)=s\left[-z_{0}+f\left(z_{0}+\frac{z}{s}\right)\right],
$$

where we choose $s=s[f]$ so that $\left|g_{1}(0)\right|=1$. Let $g_{2}(z)=g_{1}(z)$ and $s_{2}=s_{1}=1$. Generate the sequence $g_{N}(z)$ recursively by

$$
g_{N+1}(z)=s_{N+1} s_{N} g_{N-1}\left[s_{N}^{-1} g_{N}\left(s_{N+1}^{-1} z\right)\right] \text {, }
$$

and choose real $s_{N+1}>0$ to make $\left|g_{N+1}(0)\right|=1$. If the scaling conjectures $(1.16 \mathrm{c})$ and $(1.18 \mathrm{c})$ are correct, then the sequence $\left\{g_{N}\right\}$ will alternate between two limiting functions: one function when $N$ is even and another function when $N$ is odd. 
Define

$$
\theta_{e(o)}=\lim _{\substack{N \rightarrow \infty \\ \text { even (odd) }}} \arg \left[g_{N}(0)\right]
$$

let $\Delta=\frac{\theta_{0}+\theta_{e}}{2}$, and define

$$
G_{N}(z)=e^{-i \Delta} g_{N}\left(e^{i \Delta} z\right)
$$

Conjectures (1.16c) and (1.18c) yield

$$
\lim _{N \rightarrow \infty} G_{N}^{*^{N}}(z)=F_{i, c}(z) .
$$

When $z_{0} \in U$ these transformations can be carried out analytically. Using the scaling relation (1.16) we find

$$
\begin{gathered}
\lim _{N \rightarrow \infty} s_{N}=\beta=\Omega^{-1}, \\
F_{i}(z)=e^{i \pi / 2}+z .
\end{gathered}
$$

To study scaling at $z_{c}$ we start with $f(z)=e^{2 \pi i \Omega} z+z^{2}$ and change coordinates so that

$$
g_{1}(z)=\frac{1}{s} z^{2}+s p
$$

where

$$
p=\frac{e^{2 \pi i \Omega}}{2}-\frac{e^{4 \pi i \Omega}}{4}
$$

and choose $s$ as in (2.1) so that $\left|g_{1}(0)\right|=1$. Equation (2.8) has a critical point at the origin, and a fixed point with derivative $e^{2 \pi i \Omega}$. Composition of nonlinear functions generates higher powers of $z$. Thus we must carry out the transformation (2.2) on a computer, keeping many terms in the power series expansion

$$
g_{N}(z)=\sum_{m=0}^{M} A_{2 m}^{(N)} z^{2 m}
$$

After many iterations of the transformation (2.2), errors introduced by the truncation of the power series in (2.10) will ruin the convergence of $\left\{g_{N}\right\}$ to the limiting functions. We have carried out this calculation with $M=38$. We get the best convergence for $N \sim 28$; subsequent transformations drive the sequence away from the limiting functions. Taking $g_{27}(z)$ and $g_{28}(z)$ as the best odd and even limiting functions, we compute the angle $\Delta$ and obtain an approximation to $F_{c}(z)$ (Table 1). Comparing $g_{26}(z)$ and $g_{28}(z)$ we estimate the accuracy of our approximation as $10^{-5}$ inside the unit disk.

By generalizing Newton's method to function spaces we can obtain greater accuracy in $F_{c}$ without repeating the calculation (2.2) with larger $M$. First we derive a pair of equations which $F_{c}$ must obey. From the definition of Fibonacci 
Table 1. Coefficients in power series expansion of $F_{c}$, Eq. (2.10)

\begin{tabular}{llrrrrrr}
\hline$m$ & 0 & 1 & 2 & 3 & 4 & \multicolumn{1}{c}{5} & 6 \\
\hline $\operatorname{Re}\left[A_{2 m}\right]$ & 0.593 & 0.622 & -0.160 & 0.056 & -0.015 & -0.001 & 0.006 \\
$\operatorname{Im}\left[A_{2 m}\right]$ & 0.805 & -0.365 & 0.240 & -0.148 & 0.084 & -0.045 & 0.023 \\
\hline
\end{tabular}

numbers (1.12), we find two recursion relations which $f_{N}$ obeys,

$$
\begin{aligned}
& f_{N}(z)=f_{N-2} \circ f_{N-1}(z), \\
& f_{N}(z)=f_{N-1} \circ f_{N-2}(z) .
\end{aligned}
$$

Equations (2.11a) and (2.11b) are equivalent because

$$
f_{1} \circ f_{0}(z)=f_{0} \circ f_{1}(z)
$$

where

$$
f_{0}(z)=z
$$

In other words, all $f_{N}$ are iterates of the function $f$ and hence commute. Combining Eqs. (2.11) with the scaling hypothesis (1.16c) and (1.18c), we find that $F_{i}(z)$ must satisfy

$$
\begin{gathered}
F_{i}(z)=\beta^{2} F_{i}\left[\beta^{-1} F_{i}^{*}\left(\beta^{-1} z\right)\right], \\
F_{i}(z)=\beta F_{i}^{*}\left[\beta F_{i}\left(\beta^{-2} z\right)\right],
\end{gathered}
$$

and that $F_{c}$ must satisfy

$$
\begin{gathered}
F_{c}(z)=\alpha^{2} F_{c}\left[\alpha^{-1}\left[F_{c}^{*}\left(\alpha^{-1} z\right)\right],\right. \\
F_{c}(z)=\alpha F_{c}^{*}\left[\alpha F_{c}\left(\alpha^{-2} z\right)\right] .
\end{gathered}
$$

It is easy to check that Eqs. (2.14) are satisfied by the function $F_{i}(z)$ given by Eq. (2.7). Note that while the distinction between forms $a$ and $b$ of Eq. (2.11) is apparently trivial, it has nontrivial consequences in Eqs. (2.14) and (2.15).

To apply Newton's method to improve the solution of Eqs. (2.15), we introduce the operators $\mathcal{N}_{s}^{a}$ and $\mathscr{N}_{s}^{b}$

$$
\begin{gathered}
\mathscr{N}_{s}^{a}[F(z)]=s^{2} F\left[s^{-1} F^{*}\left(s^{-1} z\right)\right], \\
\mathscr{N}_{s}^{b}[F(z)]=s F^{*}\left[s F\left(s^{-2} z\right)\right] .
\end{gathered}
$$

The solution of Eqs. (2.15) is a fixed point of both $\mathscr{N}_{s}^{a}$ and $\mathscr{N}_{s}^{b}$ with $s=\alpha$. The solution of Eqs. (2.14) is a fixed point of both $\mathscr{N}_{s}^{a}$ and $\mathscr{N}_{s}^{b}$ with $s=\beta$. We show explicitly how to locate the fixed point of $\mathcal{N}_{\beta}^{a}$ because difficulties appear in this "trivial" case which we must understand before we can solve the nontrivial equations.

Assume that $G^{(0)}(z)$ is close to a fixed point of $\mathcal{N}_{\beta}^{a}$, and let $\mathbb{D} \mathcal{N}_{\beta}^{a}[G]$ be the Jacobian of $\mathcal{N}_{\beta}^{a}$ evaluated at the function $G(z)$. The sequence $G^{(N)}(z)$ defined by

$$
G^{(N)}(z)=G^{(N-1)}(z)-\left(1-\mathbb{D} \mathcal{N}_{\beta}^{a}\left[G^{(N-1)}\right]\right)^{-1} \cdot\left(G^{(N-1)}(z)-\mathcal{N}_{\beta}^{a}\left[G^{(N-1)}(z)\right]\right)
$$


converges quadratically to a fixed point, provided the Jacobian has no unit eigenvalues. To compute the Jacobian we introduce a basis for the space of analytic functions by the coefficients in the power series expansion

$$
F(z)=\sum_{m=0}^{M}\left[\psi_{F}^{(2 m)}+i \psi_{F}^{(2 m+1)}\right] z^{m} .
$$

Define the $(m, n)$ element of $\mathbb{D} \mathscr{N}[F]$ by

$$
\partial \psi_{\mathscr{N}[F]}^{(m)} / \partial \psi_{F}^{(n)} \text {. }
$$

Both $\mathbb{D} \mathcal{N}_{\beta}^{a}\left[F_{i}\right]$ and $\mathbb{D}_{\mathcal{N}_{\beta}^{b}}^{b}\left[F_{i}\right]$ are upper triangular with diagonal blocks corresponding to coefficients of $z^{m}$ taking the form

$$
\left(\begin{array}{cc}
\beta^{2(1-m)}+\beta^{1-m} & 0 \\
0 & \beta^{2(1-m)}-\beta^{1-m}
\end{array}\right) .
$$

$\mathrm{D} \mathcal{N}_{\beta}^{a}$ differs from $\mathbb{D} \mathcal{N}_{\beta}^{b}$ in off-diagonal blocks.

The Jacobian $\mathbb{D}_{\beta}^{a}\left[F_{i}\right]$ has two unit eigenvalues. The eigenvalue $\beta^{2}-\beta=1$, associated with $\psi_{F}^{(1)}$, is a consequence of the dilation symmetry of Eqs. (2.14). We can remove this symmetry by defining a new transformation on the space of functions with $|F(0)|=1$. This transformation is $N_{s[F]}^{a}$ with $s[F]$ chosen so that

$$
\left|N_{s[F]}^{a}[F(0)]\right|=1 .
$$

The second unit eigenvalue $\beta^{-2}+\beta^{-1}=1$ has no obvious interpretation. The corresponding perturbation violates Eq. (2.14b). Any function which is obtained by iteration of a single function $f$ must obey Eq. (2.14b) if it obeys (2.14a). Thus this perturbation has no physical relevance. Thus the fixed point of $N_{\beta}^{a}$ is not locally unique in the space of functions with $|F(0)|=1$. A commutativity condition

$$
\mathscr{C}(z) \equiv N_{s}^{a}[F(z)]-N_{s}^{b}[F(z)]=0
$$

is required to isolate the physical solution of Eqs. (2.14).

With these considerations we can apply Newton's method to determine $F_{c}$. Just as in the trivial case, $\mathbb{D} \mathcal{N}_{\alpha}^{a}\left[F_{c}\right]$ has two unit eigenvalues. The restriction to a space of normalized functions (2.21) removes one of these. Holding $\theta=\operatorname{Arg}[F(0)]$ fixed removes the other. Thus we define a new transformation $N_{\theta}^{\prime}$ on the set

$$
\Psi_{F}=\left\{\psi_{F}^{(2)}, \psi_{F}^{(3)}, \ldots\right\}
$$

where

$$
F(z)=e^{i \theta}+\sum_{m=1}^{M}\left[\psi_{F}^{(2 m)}+i \psi_{F}^{(2 m+1)}\right] z^{2 m} .
$$

$N_{\theta}^{\prime}$ is identical to $N_{s}^{a}$ except that $F(0)$ is held constant. Newton's method locates fixed points $\Psi_{\theta}$ of $N_{\theta}^{\prime}$ which are locally unique solutions of

$$
\Psi_{\theta}=N_{\theta}^{\prime}\left[\Psi_{\theta}\right] \text {. }
$$

As we vary $\theta$, a line of such solutions is generated.

The condition $\mathscr{C}(z)=0$ for some $z$ determines the physically relevant solution of (2.25). We choose, somewhat arbitrarily, to evaluate $\mathscr{C}$ at $z_{t}=0.5978-0.9016 i$, and 
Table 2. Approach to universal values of $\theta$ and $\alpha$ as the number of coefficients in $F_{c}$ is increased. Note that the error is proportional to $\left|\operatorname{Im}\left[\mathscr{C}\left(z_{t}\right)\right]\right|$

\begin{tabular}{llll}
\hline$M$ & $\theta$ & $\alpha$ & $\left|\operatorname{Im}\left[\mathscr{C}\left(z_{t}\right)\right]\right|$ \\
\hline 20 & 0.9356 & 1.3477 & $1.2 \times 10^{-3}$ \\
30 & 0.9361096 & 1.3478327 & $3.7 \times 10^{-6}$ \\
40 & 0.936110819 & 1.347831985 & $5.7 \times 10^{-8}$ \\
50 & 0.9361107980 & 1.3478319950 & $2.1 \times 10^{-10}$ \\
\hline
\end{tabular}

vary $\theta$ until $\left|\operatorname{Re}\left[\mathscr{C}\left(z_{t}\right)\right]\right|<10^{-12}$. We gauge the accuracy of our solution by $\operatorname{Im}\left[\mathscr{C}\left(z_{t}\right)\right]$. Table 2 lists values of $\alpha$ and $\theta$ obtained as we increase the number $M$ of coefficients in our power series representation (2.24).

\section{Universality}

We have now checked the scaling hypothesis (1.18c) numerically for the special case (2.8). How can we test the hypothesis for an entire family of mappings? We find the answer by analyzing transformations in function space. In the previous section we defined a transformation (2.2)-(2.5) with the universal function $F_{c}$ as a fixed point. In this section we study the evolution of perturbations on this function. Perturbations with eigenvalue $|\lambda|<1$ vanish under repeated transformations and are called "irrelevant" because their presence does not affect scaling. Perturbations with eigenvalue $|\lambda|>1$ grow under repeated transformations and are called "relevant" because they correspond to physical parameters which must be controlled to observe scaling. We call perturbations with eigenvalue $|\lambda|=1$ "marginal." As we have seen in the previous section it is desirable to understand the effect of marginal perturbations.

Note that the transformation (2.2) is of second order. It takes a pair of functions and produces a third. The corresponding eigenvalue problem is nonlinear and difficult to solve directly. Feigenbaum, Kadanoff, and Shenker show how to avoid this problem by defining a new transformation on a space of pairs of functions

$$
\begin{gathered}
\mathscr{R}_{s}^{a}\left[\begin{array}{l}
U(z) \\
V(z)
\end{array}\right]=\left[\begin{array}{c}
s^{2} V\left(s^{-1} U^{*}\left(s^{-1} z\right)\right) \\
U(z)
\end{array}\right], \\
\mathscr{R}_{s}^{b}\left[\begin{array}{l}
U(z) \\
V(z)
\end{array}\right]=\left[\begin{array}{c}
s U^{*}\left(s V\left(s^{-2} z\right)\right) \\
U(z)
\end{array}\right] .
\end{gathered}
$$

The transformations (3.1) are fully equivalent to the transformations (2.16). When $s=\alpha$ the pair $\left[\begin{array}{l}F_{c} \\ F_{c}\end{array}\right]$ is a fixed point, whereas $\left[\begin{array}{l}F_{i} \\ F_{i}\end{array}\right]$ is a fixed point when $s=\beta$.

To evaluate the growth or decay of perturbations we must linearize (3.1) around $F_{i}$ or $F_{c}$. Assume $F+\phi_{\lambda}$ is a fixed point function plus an eigenperturbation 
Table 3. Leading eigenvalues of Eq. (3.2). $R=$ "relevant," $M=$ "marginal," and $N=$ "noncommuting"

\begin{tabular}{llcr}
\hline $\begin{array}{l}\text { Physical } \\
\text { identification }\end{array}$ & Relevance & Eigenvalue & \\
\cline { 3 - 4 } & & Interior & Critical \\
\hline Winding number & $R$ & $-\Omega^{-2}$ & $-\Omega^{-2}$ \\
Dissipation & $R$ & $\Omega^{-2}$ & $\Omega^{-2}$ \\
& $N$ & $-\beta$ & $-\chi$ \\
Rotation & $N$ & $\beta$ & $\gamma$ \\
Dilation & $M$ & -1 & -1 \\
& $N$ & -1 & 1 \\
& $M$ & 1 & 1 \\
\hline
\end{tabular}

with eigenvalue $\lambda$. Eigenperturbations of $R$ have the form $\left[\begin{array}{c}\lambda \phi_{\lambda} \\ \phi_{\lambda}\end{array}\right]$.

$$
\left[\begin{array}{c}
F+\lambda^{2} \phi_{\lambda} \\
F+\lambda \phi_{\lambda}
\end{array}\right]=\left[\begin{array}{c}
F \\
F
\end{array}\right]+\mathbb{D} \mathscr{R}_{s}^{\mu}\left[\begin{array}{c}
\lambda \phi_{\lambda} \\
\phi_{\lambda}
\end{array}\right]
$$

when $\mu=a$ or $b$ and

$$
\begin{aligned}
\mathbb{D} \mathscr{R}_{s}^{a}\left[\begin{array}{c}
U(z) \\
V(z)
\end{array}\right] & =\left[\begin{array}{c}
s^{2} V\left(s^{-1} F^{*}\left(s^{-1} z\right)\right)+s F^{\prime}\left(s^{-1} F^{*}\left(s^{-1} z\right)\right) U^{*}\left(s^{-1} z\right) \\
U(z)
\end{array}\right], \\
\mathbb{D} \mathscr{R}_{s}^{b}\left[\begin{array}{c}
U(z) \\
V(z)
\end{array}\right] & =\left[\begin{array}{c}
s U^{*}\left(s F\left(s^{-2} z\right)\right)+s^{2} F^{*}\left(s F\left(s^{-2} z\right)\right) V\left(s^{-2} z\right) \\
U(z)
\end{array}\right] .
\end{aligned}
$$

We introduce a power series basis $\left\{\psi^{m}\right\}$ with

$$
\begin{aligned}
& \left(\psi^{(4 m)}, \psi^{(4 m+1)}, \psi^{(4 m+2)}, \psi^{(4 m+3)}\right) \\
& \quad=\left(\operatorname{Re}\left[U_{m}\right], \operatorname{Im}\left[U_{m}\right], \operatorname{Re}\left[V_{m}\right], \operatorname{Im}\left[V_{m}\right]\right) .
\end{aligned}
$$

In this basis the Jacobian at the trivial fixed point, $\mathbb{D} \mathscr{R}_{\beta}^{\mu}\left[F_{i}\right]$, is block upper triangular with diagonal blocks corresponding to coefficients of $z^{m}$ taking the form

$$
\left(\begin{array}{cccc}
\beta \beta^{-m} & 0 & \beta^{2} \beta^{-2 m} & 0 \\
0 & -\beta \beta^{-m} & 0 & \beta^{2} \beta^{-2 m} \\
1 & 0 & 0 & 0 \\
0 & 1 & 0 & 0
\end{array}\right)
$$

$\mathbb{D} \mathscr{R}_{\beta}^{a}$ differs from $\mathbb{D} \mathscr{R}_{\beta}^{b}$ in off diagonal blocks. The four eigenvalues of the $m^{\text {th }}$ block are

$$
\lambda= \pm \beta^{2-m}, \quad \pm \beta^{-m} .
$$

Table 3 summarizes the interpretations of the marginal and relevant perturbations. The eigenvalues $+\beta^{2}$ and $-\beta^{2}$ correspond, respectively, to adding a real or imaginary constant to $F_{i}$. A real constant destroys the Siegel domain by making the function (1.1) expanding or contracting at its fixed point. An imaginary 
constant changes the winding number of the Siegel domain. We can think of these perturbations as adding a constant to $\Omega$ in Eq. (1.1). A real constant changes the winding number, whereas an imaginary constant changes $\left|f^{\prime}(0)\right|$. Perturbations with eigenvalue $\pm \beta^{2-m}$ with $m \geqq 1$ violate Eq. (2.22) and hence are not contained in the physical spectrum. Perturbations with eigenvalues $\pm \beta^{-m}$ correspond to coordinate changes. To see this let

$$
\phi_{\varepsilon, m}(z)=z\left(1+\varepsilon z^{m}\right)
$$

and let

$$
F_{\varepsilon, m}(z)=\phi_{\varepsilon, m}^{-1} \circ F_{i} \circ \phi_{\varepsilon, m}(z) .
$$

From Eqs. (3.1), (3.2), and (3.8) we have

$$
\begin{aligned}
F(z)= & \left(\phi_{\lambda^{2} \varepsilon, m} \beta^{2} \phi_{\varepsilon, m}^{-1}\right) \circ F\left(\phi_{\varepsilon, m} \beta^{-1} \phi_{\lambda \varepsilon, m}^{*-1}\right) \\
& \circ F^{*}\left(\phi_{\lambda \varepsilon, m}^{*} \beta^{-1} \phi_{\lambda^{2} \varepsilon, m}^{-1}\right)(z), \\
F(z)= & \left(\phi_{\lambda^{2} \varepsilon, m} \beta \phi_{\lambda \varepsilon, m}^{*}\right) \circ F^{*}\left(\phi_{\lambda \varepsilon, m}^{*} \beta \phi_{\varepsilon, m}^{-1}\right) \\
& \circ F\left(\phi_{\varepsilon, m} \beta^{-2} \phi_{\lambda^{2} \varepsilon, m}^{-1}\right)(z) .
\end{aligned}
$$

Comparing Eqs. (3.9) with Eqs. (2.14) we see that

$$
\begin{gathered}
\phi_{\lambda^{2} \varepsilon, m}\left(\beta^{2} \phi_{\varepsilon, m}^{-1}(z)\right)=\beta^{2} z, \\
\phi_{\varepsilon, m}\left(\beta^{-1} \phi_{\lambda \varepsilon, m}^{*-1}(z)\right)=\beta^{-1} z, \\
\phi_{\lambda \varepsilon, m}^{*}\left(\beta^{-1} \phi_{\lambda^{2} \varepsilon, m}^{-1}(z)\right)=\beta^{-1} z,
\end{gathered}
$$

and

$$
\begin{gathered}
\phi_{\lambda^{2} \varepsilon, m}\left(\beta \phi_{\lambda \varepsilon, m}^{*-1}(z)\right)=\beta z, \\
\phi_{\lambda \varepsilon, m}^{*}\left(\beta \phi_{\varepsilon, m}^{-1}(z)\right)=\beta z, \\
\phi_{\varepsilon, m}\left(\beta^{-2} \phi_{\lambda^{2} \varepsilon, m}^{-1}(z)\right)=\beta^{-2} z .
\end{gathered}
$$

Using the facts that

$$
\begin{aligned}
\phi_{\varepsilon, m}(a z) & =a \phi_{a \varepsilon, m}(z), \\
\phi_{\varepsilon, m}^{*}(z) & =\phi_{\varepsilon^{*}, m}(z), \\
\phi_{\varepsilon, m}^{-1}(z) & =\phi_{-\varepsilon, m}(z),
\end{aligned}
$$

we find that $\phi_{\varepsilon, m}$ is indeed an eigenperturbation with eigenvalue $\lambda= \pm \beta^{-m}$. Thus $\varepsilon$ grows at the rate $\beta^{-m}$ when $\varepsilon$ is real and at the rate $-\beta^{-m}$ when $\varepsilon$ is imaginary. When $m=0$ these perturbations are marginal and correspond, respectively, to dilation and rotation of coordinates.

We numerically computed the spectrum of perturbations on the nontrivial fixed point $F_{c}$. Table 3 lists eigenvalues of perturbations which are analytic functions of $z^{2}$. Note that the two relevant commuting eigenvalues reproduce their trivial values. This is a consequence of Eq. (1.10); winding number is not renormalized in the Siegel theory. 
Generalizing the space of perturbations on $F_{c}$ leads to relevant eigenvalues not included in Table 3. One such perturbation translates the coordinate system,

$$
\phi(z)=z+\varepsilon .
$$

The eigenvalue of the perturbation is $\alpha$ when $\varepsilon$ is real and $-\alpha$ when $\varepsilon$ is imaginary. We have now outlined the extent of universality. In order to observe trivial scaling in $U$, we need to fix the magnitude and argument of $f^{\prime}(0)$. In order to observe nontrivial scaling, we need the additional condition

$$
z_{0}=z_{c} .
$$

Relaxing (3.13) yields either trivial scaling or chaos. We discuss this question further in the following section.

\section{Properties of $\boldsymbol{F}_{\boldsymbol{c}}$}

\section{A. Asymptotic Behavior}

In Sect. 2, we established, numerically, that Fibonacci iterates of the function (2.8) converge to a limit. In Sect. 3 we showed that this limit is universal for functions with quadratic extrema at $z_{c}$, and a fixed point nearby with derivative $e^{2 \pi i \Omega}$. We have not yet tested Manton and Nauenberg's conjecture that $\partial U$ passes through $z_{c}$. If $z_{c} \notin \partial U$, the scaling laws we have obtained have no relevance to $\partial U$. The following argument provides strong evidence that $z_{c} \in \partial U$.

If $z_{\mathrm{c}} \in \partial U$, then in any neighborhood of $z_{\mathrm{c}}$ there are points that lie on smooth Siegel curves. Consider a function in the universality class of $F_{c}$ in a coordinate system such that

$$
\lim _{N \rightarrow \infty} \alpha^{N} f_{N}^{*^{N}}\left(\alpha^{-N} z\right)=F_{c}(z),
$$

and $\operatorname{Re}[F(0)]>0$. We wish to show that in this coordinate system there is an interval $(0, \lambda)$, along the positive real axis, which is entirely contained in $U$. For $z \in U$ we have

$$
f_{N}(z)=z+\sum_{n=1}^{\infty}(-\Omega)^{n N} C_{n}(z),
$$

where $C_{n}$ are smooth functions obtained by differentiating the conjugacy function $\phi(w)$. In a generalization of a calculation performed by Manton and Nauenberg, we combine Eqs. (4.1) and (4.2) to get

$$
\lim _{N \rightarrow \infty}\left\{z+\sum_{n=1}^{\infty}(-\Omega)^{n N} \alpha^{N} C_{n}^{*^{N}}\left(\alpha^{-N} z\right)\right\}=F_{c}(z) .
$$

Assuming that the functions $C_{n}(z)$ behave like power laws for small $z$, and requiring that $(-\Omega)^{n N} \alpha^{N} C_{n}^{*^{N}}\left(\alpha^{-N} z\right)$ be independent of $N$ for each $n$, we find that $F_{c}(z)$ has the formal expansion for $\operatorname{Re}(z) \rightarrow+\infty$

$$
F_{c}(z)=z \sum_{m=0}^{\infty} A_{m} i^{m} z^{-m v}
$$


Table 4. Asymptotic behavior of $F_{c}$ compared with Eq. (4.8)

\begin{tabular}{lll}
\hline$z$ & $F_{c}(z)$ & $z\left(1+i \gamma z^{-v}\right)^{1 / v}$ \\
\hline 1.3 & $1.36077+0.517668 i$ & $1.36072+0.517603 i$ \\
1.4 & $1.45260+0.497852 i$ & $1.45254+0.497834 i$ \\
1.5 & $1.54582+0.479609 i$ & $1.54577+0.479620 i$ \\
1.6 & $1.64016+0.462807 i$ & $1.64013+0.462830 i$ \\
1.7 & $1.73542+0.447310 i$ & $1.73541+0.447334 i$ \\
1.8 & $1.83141+0.432989 i$ & $1.83142+0.433006 i$ \\
1.9 & $1.92802+0.419724 i$ & $1.92803+0.419732 i$ \\
2.0 & $2.02512+0.407405 i$ & $2.02513+0.407405 i$ \\
\hline
\end{tabular}

where

$$
v=\frac{\ln \beta}{\ln \alpha}=1.6121 \ldots
$$

and $A_{m}$ are real constants, $A_{0}=1 . F_{c}$ should take the form (4.4) if $z_{c} \in \partial U$. Conversely, if $F_{c}$ is of this form, then it is likely (though not proven) that $z_{c} \in \partial U$.

We checked this result numerically. We cannot directly evaluate our power series expansion of $F_{i}$ for $z \gtrsim 1.35$ because of singularities which are discussed in Part $\mathrm{B}$ of this section. Instead we use the fixed point equations (2.15) to analytically continue $F_{c}$ beyond its radius of convergence. We can find an analytic expression for $F_{c}$ which is in very good agreement with the numerical data. Assume we can write

$$
F_{c}(z)=h^{-1 / v}\left(z^{-v}\right),
$$

where $h$ is analytic and $h(z) \sim z$ for small $z$. Requiring that $F_{c}$ be a fixed point of $\mathcal{N}_{s}^{\mu}$ with $s=\alpha$, we find that $h$ must be a fixed point of $\mathcal{N}_{s}^{\mu}$ with $s=\Omega$. Note that the scale factor $s=\Omega$ is the inverse of the trivial scale factor $s=\beta$. Thus we can obtain a fixed point of $\mathscr{N}_{\beta}^{\mu}$ from the trivial fixed point of $\mathscr{N}_{\beta}^{\mu}$ by the coordinate change $\phi(z)=1 / z$. Thus

$$
h(z)=\frac{z}{1+i \gamma z},
$$

where $\gamma$ is an arbitrary real constant, and

$$
F_{c}(z)=z\left(1+i \gamma z^{-v}\right)^{1 / v} .
$$

We find Eq. (4.8) fits the numerical data best for $\gamma=1.013205$ (Table 4). We speculate that Eq. (4.8) is exact up to corrections which vanish faster than any power of $z^{-v}$ as $\operatorname{Re}(z) \rightarrow+\infty$.

\section{B. Singularities of $F_{c}$}

Examination of ratios of Taylor series coefficients of $F_{c}$ (Table 1) suggests that the series has a radius of convergence roughly equal to $\alpha$. We determine the precise 
location of singularities in $F_{c}$ in the following manner. Define the functions

$$
\begin{gathered}
P(z)=\alpha^{-1} F_{c}^{*}\left(\alpha^{-1} z\right), \\
Q(z)=\alpha \bar{F}_{c}\left(\alpha^{-2} z\right) .
\end{gathered}
$$

If a point $\hat{z}$ is a fixed point of either $P$ or $Q$, then Eqs. (2.15) require that $F_{c}$ must vanish or have a singularity at $\hat{z}$. Note that if $\hat{z}$ is the singularity closest to the origin, then the power series (4.9) are evaluated within their circle of convergence for $z$ in a neighborhood of $\hat{z}$. We find

$$
\hat{z}=0.14377-1.3437 i
$$

is a fixed point of both (4.9a) and (4.9b). For $z$ close to $\hat{z}$ we have

$$
\begin{gathered}
F_{c}(z)=\alpha^{2} F_{c}\left[\hat{z}+P^{\prime}(\hat{z})(z-\hat{z})\right], \\
F_{c}(z)=\alpha \bar{F}_{c}\left[\hat{z}+Q^{\prime}(\hat{z}) \overline{(z-\hat{z})}\right] .
\end{gathered}
$$

Evaluating the derivatives, we find that both $P^{\prime}(\hat{z})$ and $Q^{\prime}(\hat{z})$ are real. Thus as we approach $\hat{z}$ from a region where $F_{c}(z)$ is analytic, we expect

$$
F_{c}(z) \sim(z-\hat{z})^{-0.89099 \ldots}
$$

where the exponent is computed from the magnitude of the derivatives.

Equation (2.15) allows us to analytically continue $F_{c}$ beyond its radius of convergence. In this way we find a family of singularities in $F_{c}$, each with the same power law behavior as $\hat{z}$. Let $z$ be some point of singularity of $F_{c}$. Any point $w$ satisfying

$$
z=P(w)
$$

or

$$
z=Q(w)
$$

acquires the same singularity as $z$, provided $P$ or $\bar{Q}$ is analytic at $w$.

Figure 2 shows the locations of some singularities determined in this manner. In Fig. $2 \mathrm{a}$ the points $A_{0}$ and $B_{0}$ are defined by

$$
\begin{aligned}
& -\hat{z}=P\left(A_{0}\right), \\
& -\hat{z}=Q\left(B_{0}\right) .
\end{aligned}
$$

We obtain the points $A_{n}$ and $B_{n}$ by repeated application of $P^{-1}$. Note that the sequences $A_{n}$ and $B_{n}$ converge to $z$ creating a sharp point. We apply Eqs. (4.14) with the sets $A_{n}$ and $B_{n}$ on the left hand side to get $C_{0}$ and $D_{0}$,

$$
\begin{aligned}
& -\left\{A_{n}, B_{n}\right\}=P\left(C_{0}\right), \\
& -\left\{A_{n}, B_{n}\right\}=Q\left(D_{0}\right) .
\end{aligned}
$$

Repeated application of $P^{-1}$ generates $C_{n}$ and $D_{n}$. Each $C_{n}$ and $D_{n}$ is a conformal image of $\left\{A_{n}, B_{n}\right\}$ (Fig. 2b). We could continue this process indefinitely. Each singularity lies at the point of a scaled-down version of the entire set of singularities (Fig. 2c). 

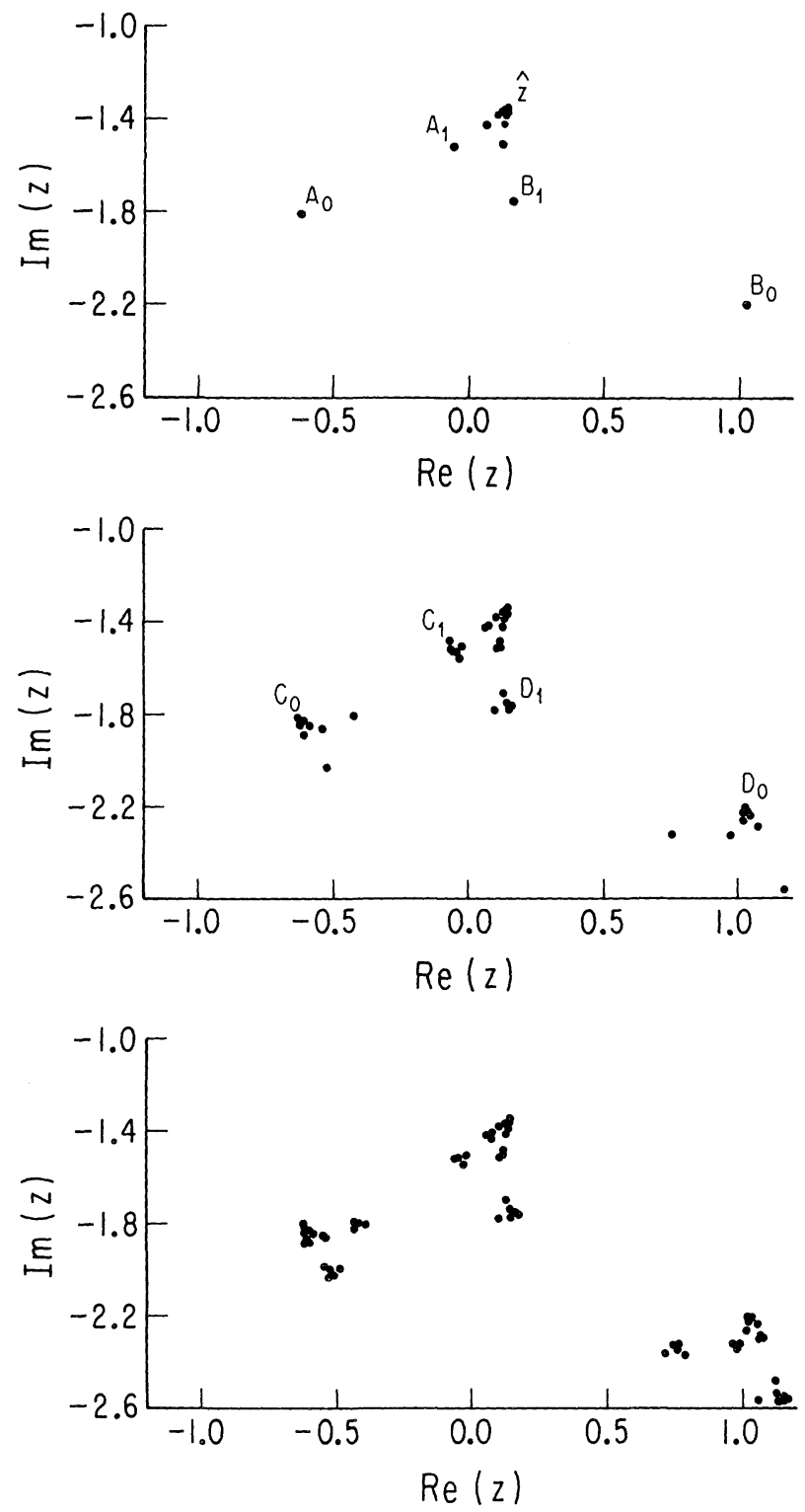

Fig. 2a-c. Singularities of $F_{c}$. a-c show the first, second, and third generation of singularities

In this way Epstein and Lascoux [17] proved the existence of a natural boundary for Feigenbaum's universal period doubling function. From Fig. 2c we cannot see whether or not the set of singularities in $F_{c}$ forms a continuous wall. The existence of a natural boundary in $F_{c}$ and its relationship to the Julia set [18] of the original mapping $f$ are unresolved questions.

Acknowledgements. I am indebted to N. Manton and M. Nauenberg for describing the results of their research to me, and to P. C. Hohenberg and the Institute for Theoretical Physics in Santa Barbara for 
their hospitality at the time this work was begun. I thank Scott J. Shenker, L. P. Kadanoff, and O. Lanford for useful conversations and comments on this paper. This work was supported in part by NSF MRL Grant No. 79-24007 and by a W. R. Harper Fellowship.

\section{References}

1. Shenker, Scott J., Kadanoff, L.P.: J. Stat. Phys. 27, 631 (1982)

2. Greene, J.M.: A method for determining a stochastic transition. J. Math. Phys. 20,1183 (1979)

3. Shenker, Scott J.: Scaling behavior in a map of a circle onto itself: empirical results. Physica 5D, 405 (1982)

4. Feigenbaum, M.J.: Quantitative universality for class of nonlinear transformations. J. Stat. Phys. 19, 25 (1978); The universal metric properties of nonlinear transformations. J. Stat. Phys. 21, 669 (1979)

5. Collet, P., Eckmann, J.-P.: Iterated maps on the interval as dynamical systems. Boston : Birkhäuser 1980

6. Kadanoff, L.P.: Scaling for a critical Kolmogorov-Arnold-Moser trajectory. Phys. Rev. Lett. 47, 1641 (1981)

7. Kadanoff, L.P. : In : Melting, localization, and chaos. Proceedings of the Ninth Midwest Solid State Theory Symposium, Kalia, R.K., Vashishta, P. (eds.). New York: North-Holland 1982

8. Escande, D.F., Doveil, F.: J. Stat. Phys. 26, 257 (1981)

9. MacKay, R.: Preprint (1982)

10. Feigenbaum, M.J., Kadanoff, L.P., Shenker, Scott J.: Quasiperiodicity in dissipative systems : a renormalization group analysis. Physica 5D, 370 (1982)

11. Rand, D., Ostlund, S., Sethna, J., Siggia, E.: Phys. Rev. Lett. 49, 132 (1982)

12. Siegel, C.L.: Ann. Math. 43, 607 (1942)

13. Moser, J.K., Siegel, C.L. : Lectures on celestial mechanics. Berlin, Heidelberg, New York: Springer 1971

14. Manton, N.S., Nauenberg, M.: Universal scaling behavior for iterated maps in the complex plane. Commun. Math. Phys. 89, 555 (1983)

15. Sullivan, D., Hubbard, J., Douady, A.: Private communications (1982)

16. Schröder, E. : Math. Ann. 3, 296 (1871)

17. Epstein, H., Lascoux, J.: Analyticity properties of the Feigenbaum function. Commun. Math. Phys. 81, 437 (1981)

18. For a review of Julia sets see Brolin, H.: Ark. Mat. 6, 103 (1965)

Communicated by O. E. Lanford

Received April 11, 1983; in revised form May 11, 1983 